\title{
Microbiota conjuntival en el preoperatorio de pacientes que se someterán a cirugía de cataratas
}

Fernando Barría von-B., Henriette Chabouty, René Moreno, Freddy Ortiz y Fernando Barría M.

\footnotetext{
Hospital Clínico Regional de Concepción Dr. Guillermo Grant Benavente, Concepción, Chile. Servicio de Oftalmología (FBvB). Asociación Panamericana de Oftalmología, Comité de Prevención de la Ceguera (FBvB) Laboratorio de Microbiología (HC). Universidad de Concepción, Facultad de Medicina, Chile. Residente de Oftalmología (RM,

Estudiante de Medicina (FBM)

Los autores declaran no tene conflictos de interés. Este trabajo no contó con fuentes de financiamiento.

Recibido: 8 de agosto de 2014 Aceptado: 6 de enero de 2015

Correspondencia a: Fernando Barría von Bischhoffshausen fdobarria@vtr.net fdobarria@gmail.com
}

\author{
Microbial flora isolated from patient's conjunctiva previous \\ to cataract surgery
}

Background. Endophtalmitis post cataract surgery is one of most feared and devastating complications resulting in serious consequences and an uncertain visual prognosis. Antimicrobial prophylaxis against endophtalmitis must be based on the best knowledge of conjuntival microbiota. Objective: To establish microbiological basis for the best antibiotic prophylaxis to prevent endophthalmitis in cataract surgery. Materials and Methods: A descriptive, cross-sectional, prospective study. A preoperative conjunctival sample was taken from the lower fornix of 118 pacients, sowing it immediately in culture media. Identification of growing colonies and susceptibility testing were performed by manual or automated methods. Results: $106(89.8 \%)$ of 118 preoperative cultures were positive. 159 bacteria were isolated in single or mixed flora, with 95\% of Gram positive organisms. Staphylococci represented $76.1 \%$ of isolated bacteria, with $82.6 \%$ of coagulase-negative staphylococci (SCN) and $17.4 \%$ of Staphylococcus aureus. Forty two percent of SCN and 38\% of S. aureus were methicillin resistan; both groups showed high susceptibility to tobramycin and fourth-generation fluoroquinolones. Conclusions: we recommend the use of topical tobramycin as pre-operative antimicrobial prophylaxis associated with povidone-iodine antisepsis. A fourth-generation quinolone is recommended when there is risk of infection.

Key words: Conjunctival bacteria, preoperative cultures, cataract surgery, endophthalmitis prevention.

Palabras clave: Bacterias conjuntivales, cultivos preoperatorios, cirugía de cataratas, prevención de endoftalmitis.

\section{Introducción}

L a cirugía de cataratas es la intervención oftalmológica más realizada en el mundo. Sin embargo, a pesar de nuestros esfuerzos, la catarata sigue siendo la principal causa de ceguera en el mundo, siendo su único tratamiento una cirugía para recuperar la visión ${ }^{1,2}$. Actualmente, 85 a $95 \%$ de los pacientes intervenidos logra recuperar la visión, lo que mejora su calidad de vida y permite reincorporarlo a la comunidad. Esto ha traído aparejado un aumento de las expectativas en los resultados visuales de la cirugía de cataratas, por lo cual una infección intraocular o endoftalmitis post-quirúrgica por colonización microbiana es una de las complicaciones post-operatorias más temidas y devastadoras con un pronóstico visual incierto, pudiendo ocasionar secuelas graves, e incluso comprometer la integridad anatómica del globo ocular, lo que se asocia a un costo sanitario elevado, y además podría derivar en problemas médico legales. La incidencia actual de esta complicación es baja, oscilando entre 0,015 y $0,5 \%{ }^{3-6}$. Sin embargo, su incidencia real puede subestimarse debido a que ciertos casos de infección bacteriana pueden confundirse con inflamación post-operatoria intensa. La fuente más importante de contaminación en la cirugía intraocular es la microbiota conjuntival $^{2,7}$. El Endophthalmitis Vitrectomy Study (EVS) reportó 69,3\% de cultivos positivos tras la toma de muestra de humor acuoso y vítreo. Las bacterias aisladas pertenecían a la microbiota de los tejidos perioculares, principalmente el grupo de Staphylococcus coagulasa negativa (Staphylococcus epidermidis y otros) presente en $70 \%$ de los cultivos, Staphylococcus aureus (10\%), Streptococcus spp (9\%), Enterococcus spp (2\%), otras especies grampositivas (3\%), y especies gramnegativas $(6 \%)^{6,8}$. La microbiota conjuntival puede ingresar a la cámara anterior, directa o indirectamente, por la manipulación quirúrgica, los instrumentales, el implante del lente intraocular, e incluso, por filtración a través de la incisión en el post-operatorio ${ }^{7,9-12}$.

Para disminuir el riesgo de las infecciones postquirúrgicas, es necesario conocer la naturaleza de esta microbiota conjuntival en nuestros pacientes, y así recomendar la mejor profilaxis antimicrobiana en cirugía de catarata $^{7,13}$, además constatar si existen factores asociados 
a infección como obstrucción lagrimal o infecciones crónicas concomitantes como blefaritis, dermatitis o conjuntivitis, uso prolongado de corticosteroides, exposición a la radiación, tabaquismo, otra cirugía ocular y exposición a la luz ultravioleta, entre otros. Para una profilaxis pre-operatoria, se recomienda, basado en la evidencia científica, el uso de povidona yodada sobre los párpados y fondos de sacos conjuntivales ${ }^{14-17}$ como una medida adicional al uso de antimicrobianos. Así mismo, se ha reportado la utilidad de las quinolonas de tercera o cuarta generación y de otros antibacterianos intracamerales como cefuroxima ${ }^{18-24}$.

La elección óptima de una antibio-profilaxis se debe basar en el espectro de cobertura bacteriana del antimicrobiano, la rapidez de acción del mismo, la duración de la acción, la penetración, su toxicidad, el costo, la microbiota del área y el patrón de susceptibilidad in vitro, entre otros ${ }^{19,20,25}$. El uso de antibacterianos tópicos en las cirugías de cataratas se recomienda para reducir temporalmente la microbiota conjuntival ${ }^{13}$, pero no hay estudios que evalúen su efecto en disminuir la incidencia de endoftalmitis, por lo cual, en este caso, su uso no es una recomendación basada en evidencia ${ }^{8}$, aunque sí es una práctica habitual. Los antimicrobianos de última generación como las fluoroquinolonas de $4^{\circ}$ generación se promocionan como profilaxis de endoftalmitis postoperatoria en cirugía de cataratas, pero su uso masivo podría generar, por presión selectiva, una resistencia antibacteriana en la microbiota de nuestros pacientes ${ }^{13,19-21}$. La guía clínica del tratamiento quirúrgico de cataratas recomienda como profilaxis peri-operatoria el uso de povidona yodada al $5 \%$ en los fondos de sacos conjuntivales, a lo menos cinco minutos previos a la intervención. (Recomendación A 1) ${ }^{1}$.

\section{Objetivo}

Establecer las bases microbiológicas para la mejor profilaxis antimicrobiana de endoftalmitis en pacientes que serán sometidos a cirugía de cataratas, en el Hospital Clínico Regional de Concepción (HCRC), a través de la identificación de la microbiota conjuntival pre-operatoria y el análisis de sus patrones de susceptibilidad in vitro.

\section{Materiales y Métodos}

Se realizó un estudio descriptivo, de corte transversal, prospectivo, en 118 pacientes con indicación de cirugía de cataratas en el Servicio de Oftalmología HCRC "Guillemo Grant Benavente", quienes cumplieron los criterios de inclusión.

\section{Criterios de inclusión}

Paciente del HCRC con indicación de cirugía de cataratas no combinada con otro tipo de cirugía oftalmológica.

\section{Criterios de exclusión}

Paciente que el día de la cirugía presenta uno o más de los siguientes factores: uso de colirios antibacterianos, enfermedad palpebral o dérmica, conjuntivitis crónica, cuadro infeccioso con tratamiento antimicrobiano en los últimos seis meses.

A los pacientes se les entrevistó, entregándoles una explicación del propósito del estudio y procedimientos involucrados, solicitando su consentimiento informado, según los Protocolos del Establecimiento. Este trabajo fue aprobado por el Comité de Ética del HCRC, según la Declaración de Helsinki.

El día de la cirugía se obtuvo una muestra conjuntival pre-operatoria en el ojo que sería intervenido. En este procedimiento se utilizó una tórula estéril. Para la recolección de la muestra se extrajo una película lagrimal desde el fondo de saco inferior y superior del ojo, previo administración de anestesia tópica y antes de administrar cualquier colirio al ojo estudiado. Las muestras fueron enviadas de inmediato al laboratorio en medio de transporte Stuart (antes de $30 \mathrm{~min}$ ). La siembra de las muestras se realizó en medios agar sangre de cordero, agar MacConkey, agar Sabouraud, caldo tripticasa en ambiente aerofílico y en agar chocolate suplementado en ambiente micro-aerofílico. Los cultivos fueron incubados a $35^{\circ} \mathrm{C}$ durante $72 \mathrm{~h}$. Todas las colonias desarrolladas se identificaron por métodos manuales convencionales y/o métodos automatizados, utilizando tarjetas NMIC/ID 92 para bacterias gramnegativas y tarjetas PMIC/ID 107 para grampositivas en el equipo Phoenix $100 \mathrm{BD}$.

La susceptibilidad in vitro fue realizada en el mismo equipo por método de microdilución y para aquellos antimicrobianos no incorporados en las tarjetas, como cloranfenicol, tobramicina y gatifloxacina, las pruebas se realizaron por método de difusión en discos en agar MuellerHinton. Ambos métodos se realizaron según las recomendaciones de la CLSI Performance Standards for Antimicrobial Susceptibility Testing del año $2013^{26}$.

Los resultados fueron ingresados al protocolo establecido, tabulado y analizado en planillas Excel.

\section{Resultados}

\section{Pacientes}

Se realizaron 118 cultivos pre-operatorios de conjuntiva en 118 pacientes ambulatorios sometidos a cirugía de cataratas, que cumplieron los criterios de inclusión, en el período entre marzo y diciembre del año 2013. Sesenta y uno de los pacientes $(51,7 \%)$ fueron mujeres, siendo el promedio de edad de 70,4 años (rango de 29 a 92 años). En trece pacientes (11\%) se contaba con el antecedente de uso de colirios (gotas) como tratamiento crónico de glaucoma y seis pacientes $(5,1 \%)$ tenían el antecedente 
Tabla 1. Distribución según número de especies recuperadas de los cultivos pre-operatorios de conjuntiva en pacientes que serán sometidos a cirugía de cataratas. Hospital Clínico Regional de Concepción, marzo a diciembre de 2013

$\begin{array}{lcc}\text { Cutivo } & \mathbf{n} & \% \\ \text { Monomicrobiano } & 65 & 55,1 \\ \text { Dos tipos de aislados } & 33 & 27,9 \\ \text { Tres o más tipos de aislados } & 8 & 6,8 \\ \text { Sin desarrollo microbiano } & 12 & 10,2 \\ \text { Total } & 118 & 100,0\end{array}$

Tabla 2. Incidencia de bacterias aisladas en cultivos pre-operatorios, de muestras conjuntivales en pacientes que serán sometidos a cirugía de cataratas. Hospital Clínico Regional de Concepción, año 2013

\begin{tabular}{|c|c|c|c|}
\hline $\mathbf{N}^{\circ}$ & Especie & $\mathbf{n}$ & $\%$ \\
\hline 1 & Staphylococcus coagulasa negativa & 100 & 62,9 \\
\hline 2 & Staphylococcus aureus & 21 & 13,2 \\
\hline 3 & Corynabacterium matruchotii & 7 & 4,4 \\
\hline 4 & Aerococcus viridans & 3 & 1,9 \\
\hline 5 & Gemella morbillorum & 2 & 1,3 \\
\hline 6 & Corynebacterium pseudodiphtheriticum & 2 & 1,3 \\
\hline 7 & Enterococcus faecalis & 2 & 1,3 \\
\hline 8 & Escherichia coli & 2 & 1,3 \\
\hline 9 & Streptococcus salivarius & 2 & 1,3 \\
\hline 10 & Micrococcus lylae & 1 & 0,6 \\
\hline 11 & Macrococcus caseolyticus & 1 & 0,6 \\
\hline 12 & Kocuria varians & 1 & 0,6 \\
\hline 13 & Gemella spp & 1 & 0,6 \\
\hline 14 & Dermabacter hominis & 1 & 0,6 \\
\hline 15 & Arcanobacterium haemolyticum & 1 & 0,6 \\
\hline 16 & Corynebacterium striatum & 1 & 0,6 \\
\hline 17 & Enterococcus gallinarum & 1 & 0,6 \\
\hline 18 & Brevundimonas versicularis & 1 & 0,6 \\
\hline 19 & Pseudomonas putida & 1 & 0,6 \\
\hline 20 & Pseudomonas aeruginosa & 1 & 0,6 \\
\hline 21 & Morganella morganii & 1 & 0,6 \\
\hline 22 & Citrobacter freundii & 1 & 0,6 \\
\hline 23 & Enterobacter aerogenes & 1 & 0,6 \\
\hline 24 & Streptococcus grupo mitis & 1 & 0,6 \\
\hline 25 & Streptococcus parasanguinis & 1 & 0,6 \\
\hline 26 & Streptococcus sanguinis & 1 & 0,6 \\
\hline \multirow[t]{2}{*}{27} & Streptococcus constellatus & 1 & 0,6 \\
\hline & Total & 159 & 100,0 \\
\hline
\end{tabular}

de hospitalización con data de más de un año previo a su cirugía programada de catarata.

\section{Cultivos}

De una muestra total de 118 cultivos pre operatorios, en 106 cultivos $(89,8 \%)$ se obtuvo un desarrollo microbiano y en 12 pacientes $(10,2 \%)$ no hubo desarrollo microbiano. Los cultivos positivos sólo presentaron aislados bacterianos. Pese a la búsqueda de hongos, en ningún paciente se obtuvo desarrollo de elementos fúngicos. De los 106 pacientes con cultivos positivos, en $65(55,1 \%)$ se desarrolló una microbiota única, en treinta y tres $(27,9 \%)$ desarrollaron microbiota doble y en ocho pacientes $(6,8 \%)$ se aisló una microbiota mixta con tres o más tipos de bacterias (Tabla 1). Todo lo anterior dio un total de 159 bacterias aisladas. Treinta y siete pacientes $(31,4 \%)$ refirieron el antecedente de diabetes mellitus.

En el grupo de 65 pacientes con un tipo de aislado, 57 bacterias pertenecían al género Staphylococcus. Sólo cuatro pacientes $(3,4 \%)$ presentaron una combinación de bacterias grampositivas y gramnegativas. A su vez cuatro pacientes presentaron aislados del género Streptococcus.

\section{Microorganismos}

En 106 cultivos (89,8\%) se desarrollaron 159 microorganismos (Tabla 2).

Del total de bacterias aisladas, $151(95 \%)$ corresponden a especies grampositivas y ocho (5\%) a gramnegativas. Del total de bacterias grampositivas, 121 (80,1\%) pertenecían al género Staphylococcus y del total de las gramnegativas, cinco $(62,5 \%)$ pertenecían a la familia Enterobacteriaceae.

$\mathrm{Al}$ analizar la frecuencia por género bacteriano, constatamos que Staphylococcus spp. representó 76,1\% del total de bacterias aisladas, constituyéndose en el género más frecuente del total de aislados. El segundo género bacteriano en frecuencia de aislamientos correspondió a Corynebacterium spp (7,5\%).

En el género Staphylococcus, 100 cepas bacterianas $(82,6 \%)$ correspondieron a Staphylococcus coagulasa negativa (SCN), cuyo principal aislado fue Staphylococcus epidermidis, y 21 (17,4\%) fueron Staphylococcus aureus (Tabla 3).

De los 118 individuos participantes, se obtuvieron 21 aislados de $S$. aureus, lo que corresponde a una prevalencia de $17,8 \%$ de portadores oculares. Así mismo, del total de pacientes participantes $31,5 \%$ presentaron diabetes mellitus (37/118) y de este grupo sólo $8,1 \%$ presentó portación ocular de $S$. aureus (3/37).

Todos los Streptococcus spp. aislados son microbiota propia de la boca (saliva y mucosas). Es importante destacar que este género representó un bajo porcentaje de aislados $(3,8 \%)$ y que, en términos generales, presentaron patrones de susceptibilidad antimicrobiana diferentes 


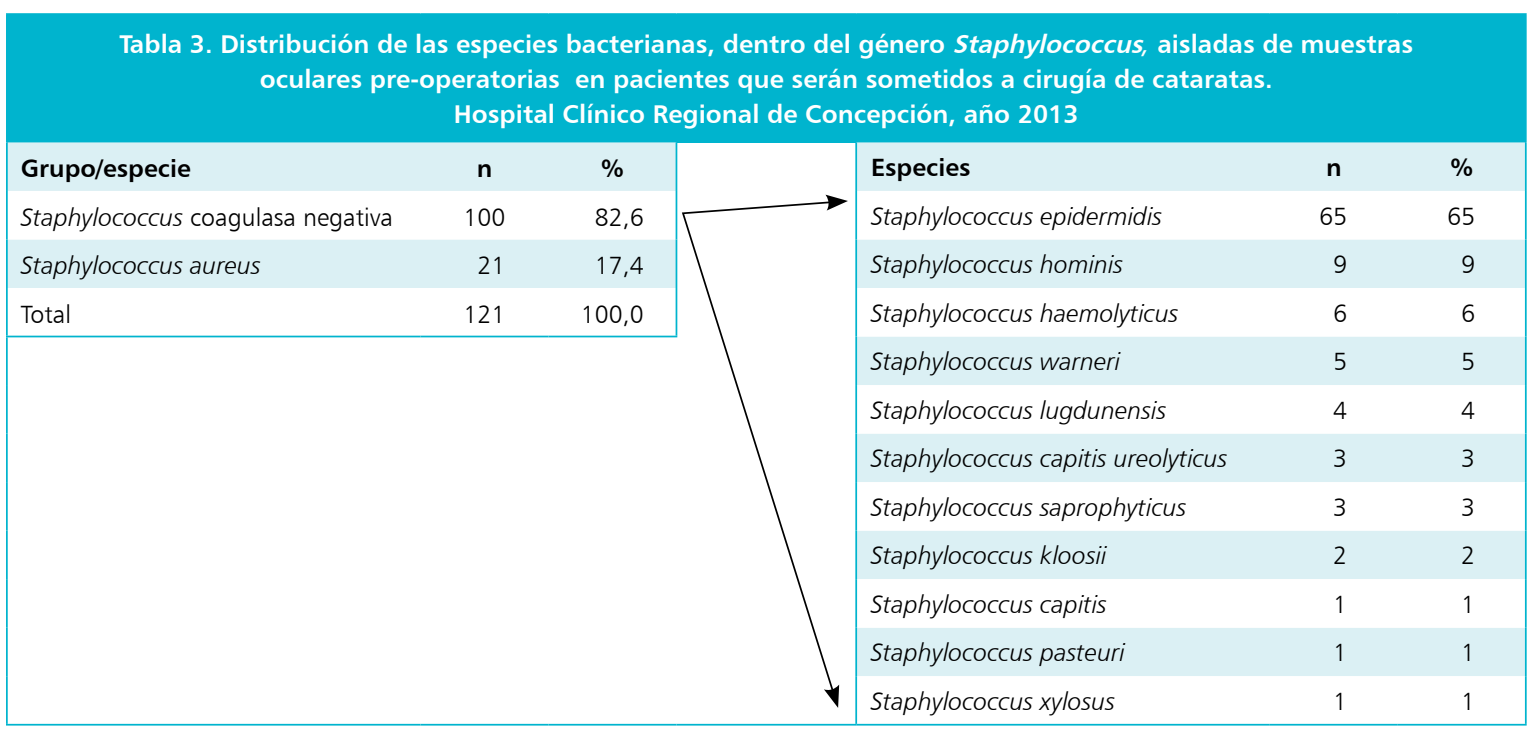

a otros géneros bacterianos, ya que por ejemplo, son constitutivamente resistentes a gentamicina. De la misma forma, la prevalencia de los aislados de bacilos no fermentadores (BNF) y Enterococcus es muy baja, favoreciendo la elección de una mejor profilaxis antimicrobiana ya que este último género es constitutivamente resistente a cefalosporinas y los BNF generalmente presentan resistencia a variados antimicrobianos.

\section{Susceptibilidad antimicrobiana}

En las siguientes tablas se muestra el perfil de susceptibilidad antibacteriana por género, haciendo énfasis en aquellos antimicrobianos que existen en el mercado como preparados clínicos oftalmológicos.

En el género Staphylococcus, SCN presentó $42 \%$ de resistencia a meticilina (SCN RM) y $S$. aureus $38,1 \%$, (SARM) (Tabla 4). Los SCN presentaron una alta susceptibilidad, mayor a $90 \%$ a fluoroquinolonas de cuarta generación y tobramicina (97\%), mostrando una aceptable susceptibilidad, mayor a $80 \%$, a ciprofloxacina (79\%), levofloxacina (79\%) y gentamicina (81\%). A su vez, todas las cepas de $S$. aureus (incluyendo SARM) presentaron $100 \%$ de susceptibilidad a tobramicina y a fluoroquinolonas de cuarta generación, una moderada susceptibilidad a cotrimoxazol $(81 \%)$ y gentamicina $(85,7 \%)$ y una baja susceptibilidad a ciprofloxacina (42,9\%). Todos los SARM presentaron una alta susceptibilidad (100\%) a vancomicina, tetraciclina, cotrimoxazol, rifampicina y resistencia a eritromicina.

Los aislados de Streptococcus del grupo viridans presentaron una alta susceptibilidad antimicrobiana $(100 \%)$ a todas las quinolonas testeadas (ciprofloxacina, levofloxacina, gatifloxacina y moxifloxacina), a cloranfenicol y vancomicina. Dentro de los antimicrobianos con
Tabla 4. Susceptibilidad in vitro de Staphylococcus aislados de muestras oculares pre-operatorias de

pacientes que serán sometidos a cirugía de cataratas. Hospital Clínico Regional de Concepción, año 2013

\begin{tabular}{|c|c|c|}
\hline Antimicrobiano & $\begin{array}{c}\text { SCN } \\
\text { (n: } 100 \text { ) } \\
\text { Sensible } \\
\%\end{array}$ & $\begin{array}{c}\text { S. aureus } \\
\text { (n: 21) } \\
\text { Sensible } \\
\%\end{array}$ \\
\hline \multicolumn{3}{|c|}{ Con presentación comercial en gotas } \\
\hline Ciprofloxacina & 79 & 42,9 \\
\hline Levofloxacina & 79 & 76,2 \\
\hline Gatifloxacina & 95 & 100 \\
\hline Moxifloxacina & 90 & 100 \\
\hline Gentamicina & 81 & 85,7 \\
\hline Tobramicina & 97 & 100 \\
\hline Cloranfenicol & 47 & 71,4 \\
\hline \multicolumn{3}{|c|}{ Sin presentación comercial oftalmológica } \\
\hline Cloxacilina & 58 & 61,9 \\
\hline Cefazolina & 58 & 61,9 \\
\hline Eritromicina & 61 & 23,8 \\
\hline Cotrimoxazol & 71 & 81,0 \\
\hline Vancomicina & 100 & 100 \\
\hline Rifampicina & 98 & 95,2 \\
\hline
\end{tabular}

preparación clínica en gotas, sólo los aminoglucósidos no presentaron buena actividad frente a este grupo bacteriano, gentamicina $(0 / 6)$ y tobramicina (4/6). Por el contrario, las cepas de Enterococcus presentaron alta susceptibilidad $(100 \%)$ a tobramicina, gatifloxacina y cloranfenicol.

Todas las enterobacterias (n: 5) presentaron susceptibilidad antimicrobiana a gentamicina, tobramicina, levofloxacina, gatifloxacina, moxifloxacina, ertapenem, 
meropenem, imipenem y frecuente susceptibilidad (4/5) a cloranfenicol, ciprofloxacina, cefotaxima, ceftazidima y cotrimoxazol. Los bacilos no fermentadores (n: 3) presentaron todas susceptibilidad a los antimicrobianos con preparación clínica de gotas (ciprofloxacina, levofloxacina, gatifloxacina, moxifloxacina, gentamicina y tobramicina), con excepción de cloranfenicol (2/3).

\section{Discusión}

La profilaxis antimicrobiana es una medida basada en evidencia que permite prevenir una infección asociada al sitio quirúrgico al reducir la microbiota en el sitio de incisión quirúrgica. Para una mejor elección en la antibioprofilaxis en cirugía de cataratas, es necesario conocer la susceptibilidad in vitro de la microbiota comensal del paciente, ya que existen antecedentes de que las infecciones del sitio quirúrgico tienen a esta microbiota como principal agente infeccioso.

En la cirugía oftalmológica, el uso de solución acuosa de povidona yodada al $5 \%$ en el saco conjuntival y al 5 ó $10 \%$ en la piel periocular es una medida universalmente aceptada para la antisepsia previa a la cirugía dejándola actuar un mínimo de tres minutos (Nivel de evidencia 2a, grado de recomendación $\mathrm{B})^{2,27}$, lo cual reduce en cerca de $90 \%$ la microbiota en el área. En pacientes alérgicos a povidona yodada se puede emplear solución acuosa de clorhexidina al $0,05 \%$. A la fecha, no se ha descrito en la literatura médica caso alguno de anafilaxia por el uso ocular de povidona yodada, pero está descrito que produce irritación o dermatitis de contacto en 1 a $4 \%$ de la población ${ }^{28}$. La povidona yodada no disminuye el riesgo de infección del sitio quirúrgico por sí sola y se recomienda que esta medida sea sumada a una profilaxis antibacteriana ${ }^{7}$. Existen diferentes guías metodológicas propuestas para la antisepsia pre-operatorio y todas ellas incluyen povidona yodada ${ }^{2,7,29}$. Los antimicrobianos tópicos pre-operatorios disminuyen el número de bacterias en la superficie ocular ${ }^{30}$, pero no se ha demostrado que ello, por sí solo, reduzca la incidencia de endoftalmitis. Debido a que la herida operatoria en esta cirugía tarda varios días en cerrarse, algunos autores incluyen el uso de gotas antibacterianas post-operatorias, pero esta medida no ha demostrado tener mayor incidencia en reducir las tasas de infección. Los cirujanos suecos han demostrado, desde $1996^{24}$, que el uso de cefuroxima intracameral $(1 \mathrm{mg})$ es efectivo. Un ensayo clínico prospectivo y aleatorio confirmó una reducción de cinco veces el riesgo de presentar una endoftalmitis post-operatoria (Nivel de evidencia 1b, grado de recomendación $\mathrm{A})^{3,30}$. En nuestro hospital no hay gotas de povidona yodada en presentación oftalmológica, aunque existe nuestra recomendación que se adquiera para el uso asociado a la cirugía de catarata. Por protocolo, como profilaxis, se usa gotas de ciprofloxacina media hora antes de la cirugía. Por otra parte, la indicación a nuestros pacientes de usar gotas tópicas del mismo antibacteriano, en el post-operatorio, no siempre es cumplida de manera que nos asegure la cobertura antimicrobiana necesaria hasta el cierre de la herida quirúrgica.

Nuestro estudio mostró un porcentaje de $89,8 \%$ de cultivos microbianos exitosos, lo que se asemeja a otros reportes $^{6-8}$. Los pacientes presentaron una microbiota conjuntival dominada por Staphylococcus spp (76,1\%), hallazgo considerado normal para la conjuntiva, indicando que la mayoría de nuestros pacientes presentan buenas condiciones higiénicas y que no son portadores de especies habitualmente patógenas. No se aislaron hongos aunque se efectuó una búsqueda dirigida. En 34,7\% de los pacientes se recuperó microbiota mixta, sólo asociada a personas mayores de 65 años, sin otro factor de riesgo. Siete pacientes $(5,9 \%)$ presentaron portación de bacilos gramnegativos, todos potencialmente patógenos y cuatro pacientes $(3,4 \%)$ presentaron portación de Streptococcus spp., lo cual demuestra la relevancia de la preparación con antiséptico. Un estudio efectuado en México arrojó valores cuatro veces mayores de portación de bacilos no fermentadores y el doble en frecuencia de portación de enterobacterias $^{31}$.

El grupo de SCN presentó $42 \%$ de resistencia a meticilina y en $S$. aureus fue de $38,1 \%$. Llama la atención el porcentaje de pacientes portadores de SAMR en su tejido conjuntival, proviniendo de la comunidad. Este porcentaje podría explicarse por el perfil de los pacientes estudiados, ya que aunque eran pacientes ambulatorios, en su mayoría eran mayores de 65 años (promedio 70,4 años) y, si bien es cierto no referían hospitalizaciones recientes -la más cercana databa a un año en 5,1\% del grupo- son pacientes que usualmente se atienden en Centros ambulatorios de Salud, es decir, se encuentran en contacto frecuente con personal de salud y este entorno en la atención. Se ha reportado que los pacientes diabéticos presentan mayor portación de $S$. aureus $^{32}$, lo que no fue constatado en nuestro estudio, sólo $8,1 \%$ de ellos presentaron portación ocular.

Varios estudios confirman que el grupo de SCN es la causa más frecuente de endoftalmitis post-operatoria, lo cual debe considerarse al momento de elegir un antimicrobiano profiláctico. En nuestro estudio, ciprofloxacina, que por norma es usado como profilaxis en nuestro hospital, no mostró una actividad adecuada sobre Staphylococcus. Las quinolonas de cuarta generación tienen un buen espectro de actividad pero, por la presión selectiva derivada de su uso, podrían favorecer la resistencia bacteriana ${ }^{33-37}$.

Dentro de los aislados no Staphylococcus encontramos bacterias de la piel (Corynebacterium spp), de la microbiota bucal (Streptococcus spp), microbiota ambiental (no fermentadores), intestinal y genitourinaria (enterobacterias y Enterococcus spp). Nuestro grupo 
recomienda que esta microbiota, pudiera ser reducida o eliminada con una buena higiene pre-operatoria sumada al uso de povidona yodada.

Es importante conocer la microbiota asociada a nuestros pacientes pero existen otros factores como el tamaño del inóculo, la virulencia del microbio, la respuesta inmunitaria del hospedero y la cicatrización de las heridas que también tienen un papel a considerar.

Para la mejor elección de una profilaxis antimicrobiana es fundamental conocer además, cómo se libera el antibacteriano en los tejidos o espacios del ojo, y cómo afectan las concentraciones de antimicrobiano a la erradicación microbiana. La acción antibacteriana en el ojo está relacionada con las concentraciones alcanzadas por el antimicrobiano en el sitio diana, así como la duración de las concentraciones eficaces durante un período de tiempo. Existen pocos estudios farmacocinéticos y farmacodinámicos de la profilaxis antibacteriana ocular; además, estos resultados se deben combinar con una comprensión de las definiciones estándares de laboratorio de "sensibilidad" o "resistencia" microbiana, aunque estos estándares de laboratorio todavía no se han adaptado a las condiciones propias del ojo.

Las gotas antibacterianas tópicas se instilan a concentraciones muy superiores a las utilizadas en procedimientos de laboratorio estándares que definen la resistencia o la sensibilidad bacteriana. No obstante, después de la instilación en el ojo, estas concentraciones se diluyen inmediatamente en la película lagrimal y experimentan una rápida eliminación por drenaje naso-lagrimal; así las concentraciones del antimicrobiano dentro del globo ocular son menores a las obtenidas en las lágrimas.

Puesto que estas concentraciones de antibacteriano instilado son muy altas en comparación con las CIM de las bacterias encontradas, se supone que las bacterias son destruidas en la superficie ocular después de la administración de las gotas antibacterianas. No obstante, esta suposición pasa por alto el importante elemento del tiempo, ya que los efectos bactericidas no suelen ser instantáneos, sino que requieren un período de contacto entre el microorganismo y el fármaco para producir un efecto bactericida. Existe estudios que demuestran que podría ser necesario un período de "tiempo de contacto" sorprendentemente mayor para erradicar incluso las cepas comunes de bacterias implicadas en la endoftalmitis post-operatoria.

En base a los perfiles de susceptibilidad antimicrobiana presentados, consideramos que como antimicrobiano tópico, tobramicina cumpliría un mejor papel profiláctico considerando el escaso arsenal antibacteriano en preparados oftalmológicos. Además, estos perfiles muestran una alta tasa de resistencia a ciprofloxacina y, es posible que dada la tendencia actual de usar en forma masiva las quinolonas de cuarta generación en preparados oftalmo- lógicos, por presión selectiva se pueda generar resistencia a estos antimicrobianos.

Estudios en humanos ${ }^{38}$ muestran que la administración tópica de tobramicina al $0,3 \%(3.000 \mu \mathrm{g} / \mathrm{ml})$ logra una rápida penetración del fármaco en el humor acuoso, con un pico de $3,24 \mu \mathrm{g} / \mathrm{ml}$ a las dos horas, presentando concentraciones terapéuticas adecuadas durante $6 \mathrm{~h}$. Estos estudios muestran además que las concentraciones alcanzadas post administración están sobre la CIM para $S$. aureus y $P$. aeruginosa después de 15 min y sobre la CIM para $E$. coli después de $30 \mathrm{~min}$, siendo éste el plazo para su administración antes de la cirugía ocular.

En la guía de ESCRS 20137, se menciona el uso de cefuroxima y fluoroquinolonas, como profilaxis antimicrobiana pre-operatoria, aun cuando no son antimicrobianos anti estafilocóccicos de primera elección; juegan a su favor las altas concentraciones que alcanzarían en una instilación ocular. Esperamos implementar en nuestro servicio la administración de antimicrobianos intracameral al finalizar la cirugía, siendo cefuroxima, nuestra recomendación dada la evidencia de bajas tasas de endoftalmitis post operatoria mostrada por los oftalmólogos suecos. La realidad nacional e institucional muestra que las cepas del género Staphylococcus son $100 \%$ susceptibles a vancomicina $^{39}$, pero este antibacteriano debe reservarse para el tratamiento de los casos de endoftalmitis con diagnóstico confirmado. Sin embargo, queremos enfatizar que lo más importante de la profilaxis es el uso de un antiséptico como povidona yodada al 5\% y aislar los folículos pilosos de las pestañas para evitar la contaminación ocular.

La posible portación de microbiota patógena podría ser evaluada efectuando un análisis de factores del paciente, descritos por estar asociados a infección, como son la presencia de una vía lagrimal impermeable, una conjuntivitis o blefaritis infecciosa, así como una dermatitis rosácea, diabetes mellitus o una hospitalización reciente. Se ha descrito como factor de riesgo para desarrollar una endoftalmitis, la presencia de complicaciones operatorias como la ruptura de la cápsula posterior que altera la anatomía ocular ${ }^{8}$.

Podemos concluir que no es necesario realizar cultivo a todos los pacientes que se someten a cirugía de cataratas, ya que basta con una profilaxis basada en estudios previos de la microbiota prevalente y de sus patrones de susceptibilidad antimicrobiana. Podría ser recomendable realizar cultivos pre-operatorios en pacientes con los factores asociados a infección ya descritos. Adherimos a las guías nacionales e internacionales y recomendamos el uso de povidona yodada en el pre-operatorio, con un tiempo mínimo de acción de tres minutos. De optar por el uso de gotas como antibio-profilaxis, recomendamos el uso de tobramicina para nuestros pacientes, aunque en un segundo estudio se podría evaluar la efectividad profiláctica de povidona versus antibioterapia tópica, tomando cultivos previo y posterior al tratamiento. 
El desafío final es reducir al mínimo la posibilidad de una infección intraocular en el post-operatorio de una cirugía de cataratas, conociendo la microbiota de la conjuntiva y su susceptibilidad antimicrobiana para orientar una antibio-profilaxis.

Con este estudio, los autores formulamos las siguientes recomendaciones clínicas al margen de conflictos de interés o financiamiento otorgado por la industria farmacéutica.

\section{Recomendaciones}

- Conocer la microbiota conjuntival y su susceptibilidad antimicrobiana en la comunidad nativa, que no esté bajo efecto de antibacterianos ni hospitalizada, para orientar la profilaxis.

- No es necesario realizar cultivo a todos los pacientes que se someten a cirugía, ya que basta con una profilaxis basada en estudios previos del tipo de microbiota prevalente.

- Realizar cultivos pre-operatorios del fondo de saco conjuntival superior e inferior en pacientes con factores de riesgo como la presencia de una blefaritis o conjuntivitis crónica o dermatitis tipo rosácea, en especial en pacientes con ojo único.

- Adherimos a las guías internacionales y recomendamos el uso de povidona yodada al $5 \%$ en el fondo del saco conjuntival, en el pre-operatorio, con un tiempo mínimo de acción de tres minutos.

- De optar por el uso de gotas como antibio-profilaxis, recomendamos el uso de tobramicina, treinta minutos antes de la incisión quirúrgica.

\section{Resumen}

Introducción: La endoftalmitis post-cirugía de cataratas es una de las complicaciones post-operatorias más temidas y devastadoras, pudiendo ocasionar secuelas graves, con un pronóstico visual incierto. La profilaxis antimicrobiana de esta complicación debe basarse en el conocimiento acabado de la microbiota presente en el territorio conjuntival. Objetivo: Establecer bases microbiológicas para una mejor profilaxis antimicrobiana de la endoftalmitis en cirugía de cataratas. Materiales y Métodos: Estudio descriptivo, de corte transversal, prospectivo. A 118 pacientes se les tomó muestra conjuntival pre-operatoria del fondo de saco inferior, sembrándola de inmediato en medios de cultivo. Las colonias desarrolladas se identificaron por métodos manuales y método de microdilución y difusión en disco. Resultados: De 118 cultivos pre-operatorios, 106 $(89,8 \%)$ desarrollaron colonias bacterianas. Se aislaron 159 bacterias conjuntivales como especie única o cultivo mixta, siendo 95\% grampositivas. El género Staphylococcus representó 76,1\% del total de bacterias aisladas, siendo $82,6 \%$ Staphylococcus coagulasa negativa (SCN) y $17,4 \%$ Staphylococcus aureus. El $42 \%$ de los SCN y $38 \%$ de $S$. aureus presentaron resistencia a meticilina, presentando ambos buena susceptibilidad a tobramicina y fluoroquinolonas de cuarta generación. Conclusiones: Considerando nuestros resultados, recomendaríamos a nuestros pacientes tobramicina tópica como antibioprofilaxis, asociada a povidona yodada como antiséptico. Una quinolona de cuarta generación podría usarse cuando existan factores de riesgo de infección.

\section{Referencias bibliográficas}

1.- Ministerio de Salud. Guía Clínica Tratamiento Quirúrgico de Cataratas Congénitas y Adquiridas. Santiago: MINSAL, 2010. http:// web.minsal.cl/portal/url/item/7220f6b9b0044 176e04001011f0113b7.pdf (accedido el 6 de agosto de 2014).

2.- Barría von B F, Meza P. Proyecto de catarata en Chile: análisis del éxito de una estrategia de salud pública, un logro de la oftalmología nacional. Arch Chil Oftalmol 2008; 65 (1): 55 62.

3.- Barry P, Cordovés L, Gardner S. ESCRS Guidelines for Prevention and Treatment of Endophthalmitis Following Cataract Surgery: Data, Dilemmas and Conclusions. 2013. http://www.escrs.org/endophthalmitis/ guidelines/ENGLISH.pdf

4.- Lundstrom M, Wejde G, Stenevi U, Thorburn W, Montan P. Endophthalmitis after cataract surgery: a nationwide prospective study evaluating incidence in relation to incision type and location. Ophthalmology 2007; 114 (5): 866-70. Epub 2007 Feb 26.

5.- Khan R I, Kennedy S, Barry P. Incidence of presumed postoperative endophthalmitis in Dublin for a 5-year-period (1997-2001). J Cataract Refract Surg 2005; 31: 1575-81.

6.- Taban M, Behrens A, Newcomb R L, Nobe M Y, Saedi G, Sweet P M, et al. Acute endophthalmitis following cataract surgery: a systematic review of the literature. Arch Ophthalmol 2005; 123: 613-20.

7.- Guía de la ESCRS para la prevención y tratamiento de la endoftalmitis después de la cirugía de catarata: datos, dilemas y conclusiones 2013. http://www.escrs.org/ endophthalmitis/guidelines/SPANISH.pdf (accedido el 6 de agosto de 2014).

8.- West E S, Behrens A, McDonnell M J, Tielsch J M, Schein O D. The incidence of endophthalmitis after cataract surgery among the U.S. Medicare population increased between
1994 and 2001. Ophthalmology 2005; 112 : 1388-94.

9.- Bausz M, Fodor E, Resch M D, Kristóf K. Bacterial contamination in the anterior chamber after povidone-iodine application and the effect of the lens implantation devic. J Cataract Refract Surg 2006; 32: 1691-5.

10.- Srinivasan O, Sambath T, Kanungo T, Kanakasabai N M. Microbial contamination of the anterior chamber during phacoemulsification. J Cataract Refract Surg 2002; 28: 2173-6.

11.- Packard R, Olson R J, Donnenfeld E D, Aisinoff S, Mah F R, O’Brien T B, et al. Endophthalmitis after cataract surgery. J Cataract Refract Surg 2006; 32: 28-35.

12.- Miller J J, Scott I U, Flynn H W, Smiddy W E, Miller D. Acute onset endophthalmitis after cataract surgery (2000-2004): Incidence, clinical settings and visual acuity outcomes after treatment. Am J Ophthalmol 2005; 139: 983-7.

13.- Ta C N, Chang R T, Singh K, Egbert P R, 
Shriver E M, Blumenkranz M S, et al. Antibiotic resistance patterns of ocular bacteria flora. Ophthalmology 2003; 110: 1946-51.

14.- Nentwich M M, Rajab M, Ta CN, He L, Grueterich M, Haritoglou C, et al. Application of $10 \%$ povidone iodine reduces conjunctival bacterial contamination rate in patients undergoing cataract surgery. Eur J Ophthalmol 2012 (4): 541-6.

15.- Quiroga L P, Lansingh V, Laspina F, Samudio M, Stanley J, Kaspar H M, et al. A prospective study demonstrating the effect of 5\% povidone-iodine application for anterior segment intraocular surgery in Paraguay. Arq Bras Oftalmol 2010; 73 (2): 125-8.

16.- Halachimi-Eyal O, Lang Y, Keness Y, Miron D. Preoperative topical moxifloxacin $0.5 \%$ and povidone-iodine $5.0 \%$ versus povidone-iodine $5.0 \%$ alone to reduce bacterial colonization in the conjunctival sac. J Cataract Refract Surg 2009; 35 (12): 2109-14.

17.- Carrim Z I, Mackie G, Gallacher G, Wykes $\mathrm{W} \mathrm{N}$. The efficacy of $5 \%$ povidoneiodine for 3 minutes prior to cataract surgery. Eur J Ophthalmol 2009; 19 (4): 560-4.

18.- García-Sáenz MC, Arias-Puente A, Fresnadillo-Martínez M J, Carrasco-Font C. Human aqueous humor levels of oral ciprofloxacin, levofloxacin, and moxifloxacin. J Cataract Refract Surg 2001; 27: 1969-74.

19.- Ta Ch N, Egbert P R, Singh K, Shriver E M, Blumenkranz M S, Miño de Kaspar H. Prospective randomized comparison of 3-day versus 1-hour preoperative ofloxacin prophylaxis for cataract surgery. Ophthalmology 2002; 109: 2036-41.

20.- Mendívil S A, Mendívil M P. The effect of topical povidone iodine, intraocular vancomycin, or both on aqueous humor cultures at the time of cataract surgery. Am J Ophthalmol 2001; 131: 293-300.

21.- Ramon G C. Caparas C G, Bolinao J G. Safety of prophylactic intracameral moxifloxacin $0.5 \%$ ophthalmic solution in cataract surgery patients. J Cataract Refrac Surg 2007; 33: 63-8.
22.- Ong-Tone L, FRCSC. Aqueous humor penetration of gatifloxacin and moxifloxacin eye drops given by different methods before cataract surgery. J Cataract Refract Surg 2007; 33: 5962.

23.- Miño de Kaspar H, Chang R T, Singh K, Egbert M R, Blumenkranz M S, Ta Ch N Prospective randomized comparison of 2 different methods of $5 \%$ povidone-iodine applications for anterior segment intraocular surgery. Arch Ophthalmol 2002; 123: 161-5.

24.- Beigi B, Westlake W, Chang B, Marsh C, Jacob J, Riordan T. The effect of intracameral, per-operative antibiotics on microbial contamination of anterior chamber aspirates during phacoemulsification. Eye 1998; 12: 390-4.

25.- Recchia F M, Busbee B G, Pearlman R B, Carvalho-Recchia C, Ho AC. Changing trends in the microbiologic aspects of post cataract endophthalmitis. Arch Ophthalmol 2005; 123 : 341-6.

26.- Clinical and Laboratory Standards Institute (CLSI), Performance Standards for Antimicrobial Susceptibility Testing; TwentyThird Informational Supplement M100-S23, January 2013

27.- Speaker M G, Menikoff J A. Prophylaxis of endophthalmitis with topical povidone-iodine. Ophthalmology 1991; 98: 1769-75.

28.- Flynn H W. Preoperative management of patients with reported povidone-iodine or penicillin/cefalosporin allergies. AAO Subespecialty Day 2010. Retina Pg 65-8.

29.- Endoftalmitis infecciosa. Guías de Práctica Clínica de la SERV 2011. https://www.serv. es/pdf/Guia_SERV_07.pdf. (accedido el 6 de agosto de 2014).

30.- Montan P G, Wejde G, Koranyi G, Rylander M. Prophylactic intra-cameral cefuroxime. Efficacy in preventing endophthalmitis after cataract surgery. J Cataract Refract Surg 2002; 28: 977-98.

31.- Córdova T, Tenorio G, García M, Miño H.
Determinación de la flora bacteriana conjuntival en el preoperatorio de la cirugía de catarata. Rev Médica Hosp General México 2008; 78 (2): 77-82.

32.- Fernández-Rubio M E, Rebolledo-Lara L, Martínez García L, Alarcón-Tomas M, Cortés Valdés C. The conjunctival bacterial pattern of diabetics undergoing cataract surgery. Eye 2010; 24: 825-34.

33.- Miyanaga M, Nejima R, Miyai T, Miyata K, Ohashi Y, Inoue Y, et al. Changes in drug susceptibility and the quinolone resistance determining region of Staphylococcus epidermidis after administration of fluoroquinolones J Cataract Refract Surg 2009; 35: 1970-8.

34.- Vasavada A R, Gajjar D, Raj S M, Vasavada V, Vasavada V. Comparison of 2 moxifloxacin regimens for preoperative prophylaxis: Prospective randomized triple-masked trial Part 2: Residual conjunctival flora. J Cataract Refract Surg 2008; 34: 1383-8.

35.- Miño de Kaspar H, Kreutzer T C, AguirreRomo I, et al. A prospective randomized study to determine the efficacy of preoperative topical levofloxacin in reducing conjunctival bacterial flora. Am J Ophthalmol 2008; 145: 136-42.

36.- He L, Ta CN, Miño de Kaspar H. Prospective randomized comparison of 1 day and 3 day application of topical $0,5 \%$ moxifloxacin in eliminating preoperative conjunctival bacteria. J Ocul Pharmacol Ther 2009; 25: 373-8.

37.- Moss J M, Sanislo S R, Ta C N. A prospective randomized evaluation of topical gatifloxacin on conjuntival flora in patients undergoing intravitreal injections. Ophthalmology 2009; 116: $1498-501$

38.- Desai S. Ocular pharmacokinetics of tobramycin A comparative radioimmunoassay and microbiological assay. Intern Ophthalmol 1993; 17: 201-10.

39.- Cifuentes M, Silva F, García P, Bello H, Briceño I, Calvo M, et al. Susceptibilidad antimicrobiana en Chile 2012. Rev Chilena Infectol 2014; 31 (2): 123-30. 